\title{
Meditations on Bowel Preps
}

\author{
Jonathan Belsey
}

Published online: 29 June 2013

(c) Springer Science+Business Media New York 2013

The randomized, controlled trial presented by Arya et al. [1] in this issue of the journal evaluates a novel approach to bowel preparation involving the ingestion of large volumes of lukewarm saline combined with a regimen of relaxation, meditation, yogic postural changes, and breathing exercises (the Shudh model), versus ingesting 2L of a PEG-based solution with bisacodyl. The Shudh model challenges existing approaches to bowel cleansing, in that it is not primarily based on the use of a laxative preparation but rather endeavours to use a number of strategies intended to trigger parasympathetic activation to encourage bowel emptying. Although the use of saline or other balanced electrolyte solutions for bowel cleansing has been evaluated in the past, the use of a multiple bolus approach (large volumes taken intermittently) appears to be novel, and may well offer unique advantages. The combination of this with yogic techniques is said by the authors to further enhance efficacy, although this component does not seem to have been evaluated in isolation in the published literature. Given the nature and unfamiliarity of this approach, the authors needed to demonstrate not only that the Shudh model offers acceptable levels of bowel cleansing, but also that it is practical and deliverable in the context of a busy clinical practise performing multiple colonoscopies.

The first objective-adequate bowel cleansing-is central to the delivery of an effective screening and diagnostic program. Diagnostic yield is inversely proportional to the adequacy of bowel cleansing in screening populations, particularly for polyp detection [2], with inadequate cleansing associated with missed diagnoses with a

J. Belsey $(\bowtie)$

The Old Brickworks, Chapel Lane, Little Cornard,

Sudbury CO10 5JU, UK

e-mail: jbelsey@jbmedical.com consequent need for repeat procedures, incurring excess risk for the patient and cost penalties for the healthcare purchaser [3]. Over the past 35 years, numerous reports of preparative methods employing a broad range of laxative agents and dosing strategies aimed at optimizing cleansing suggest that adequate or better bowel preparation is achievable in $>90 \%$ of patients [4]. Beyond the achievement of mucosal visibility, however, patient acceptability should also be considered. The burdensome nature of bowel preparation with its potential for unpleasant (and potentially serious) adverse effects is a significant factor in the willingness of the patient to undergo further colonoscopy-indeed more so than the impact of the procedure itself or any associated discomfort [5].

In the study by Arya et al. [1] a single endoscopist, blinded to the bowel preparation allocation, assessed cleansing in 133 patients undergoing colonoscopy ( $42 \%$ for screening and $58 \%$ for diagnostic purposes) using a segmental assessment scale developed by themselves for this study. Based on this scale, they demonstrated that successful cleansing (as defined below) was achieved in $97 \%$ of patients using 2L PEG with bisacodyl and $91 \%$ of those using the Shudh approach-a difference that was consistent with non-inferiority for Shudh. The result for PEG seems surprisingly high, given that treatment was given as a single dose the night before colonoscopy, an approach that is now considered to be inferior to split dose treatment [6]. Data collected for a recent meta-analysis would suggest that success rates of 70-90\% are more likely to be achieved for a regimen using low volume PEG with bisacodyl [4]. One must therefore call into question whether the cleansing assessment tool used was sufficiently discriminatory to detect truly adequate bowel preparation in this study.

The tool used for the evaluation involved the rating of six anatomical segments of the colon (rectum, sigmoid, 
descending colon, transverse colon, ascending colon and cecum) on the 5-point Arya bowel prep scale [1]. Success in this study (grade A or B) was based on achieving a score of $>12$ out of a possible range of $0-24$. This relatively low threshold lays the scoring system open to inappropriate inclusion of patients in the "successful" group-low scores in one to two segments can effectively be masked by higher scores in other segments, yielding a net "successful" score. This problem is not unique to the scale developed by Arya et al.- the same potential problem exists with other widely used scales like the Boston bowel preparation scale [7]. If one adopts a more stringent success measure, such as achievement of grade A alone, the results look more realistic, albeit less compelling for Shudh, i.e. PEG with bisacodyl achieving a success rate of 72 versus $57 \%$ for Shudh. One must be cautious when interpreting post hoc analyses of study data using different outcome measures from those specified in the protocol, but nonetheless this undoubtedly raises questions regarding the likely effectiveness of this strategy in clinical practise.

The second issue to be considered regarding the Shudh method relates to its practical application. Because it requires a degree of physical flexibility to carry out the yoga postures, it will not be applicable for all, while the sodium load associated with the saline intake may create electrolyte imbalance, especially in older patients. As a consequence, in addition to a range of disease-related exclusion criteria common to most studies of this type, patients were also not eligible for participation if they were aged $>70$ or had a history of hypertension, diabetes or osteoarthritis of the spine, shoulders, hips or knees. Given that the majority of colonoscopies globally are carried out in an older screening population, this effectively excludes a large number of patients. The authors state that $\sim 70 \%$ of those undergoing colonoscopy would not be eligible for Shudh. It is not clear whether these exclusion criteria are absolute contraindications to the procedure, or whether this simply reflects a cautious approach to study inclusion. If the latter, it would be very interesting to see the results in an older population more reflective of the population undergoing colonoscopy as a whole.

The issue of age is particularly important for the Shudh method. Existing bowel preparations are limited by issues of patient acceptability and the risk of adverse eventsparticularly biochemical disruption. As the Shudh model appears to have a relatively modest effect on biochemical variables (principally a small rise in serum sodium) and a high degree of patient acceptability and willingness to repeat, it would appear to be of particular potential value in older patients, who tend to be the most affected by existing bowel preparation regimens.

A further issue that needs exploring is the level of input needed to ensure that the regimen is properly implemented outside the context of a clinical trial. In this study, a specially trained nurse briefed the patients, who were then given a DVD demonstrating the techniques [1]. Clearly, this is somewhat more complex than the instruction required for conventional bowel preparation regimens, which themselves suffer from problems of incomplete adherence. This constitutes an important potential source of suboptimal results.

Overall, this study demonstrates sufficient levels of efficacy from the Shudh approach to warrant further exploration of the method. Although the authors present a plausible explanation for its mode of action, this remains largely speculative; a greater understanding of the relative contribution of the components of the regimen towards achieving optimal cleansing may facilitate methodological fine-tuning. Expanding screening programs expose increasingly large numbers of patients to unpleasant and potentially hazardous regimens that are far from problemfree. Any effort to produce a cleansing method that is wellaccepted by patients without sacrificing efficacy is to be applauded. Depending on the results of future research, the Shudh approach may offer a viable alternative, at least to a proportion of patients.

Acknowledgments J.B. has provided consultancy, data analytical and writing services to Norgine Ltd over the past 5 years.

Conflict of interest None.

\section{References}

1. Arya V, Gupta KA, Valluri A, Arya S, Lesser ML. Rapid colonoscopy preparation using bolus lukewarm saline combined with sequential posture changes-a randomized controlled trial. Dig Dis Sci. (Epub ahead of print). doi:10.1007/s10620-0132598-9.

2. Froehlich F, Wietlisbach V, Gonvers JJ, et al. Impact of colonic cleansing on quality and diagnostic yield of colonoscopy: the European Panel of Appropriateness of Gastrointestinal Endoscopy European multicenter study. Gastrointest Endosc. 2005;61: 378-384.

3. Rex DK, Imperiale TF, Latinovich DR, Bratcher LL. Impact of bowel preparation on efficiency and cost of colonoscopy. Am J Gastroenterol. 2002;97:1696-1700.

4. Belsey J, Crosta C, Epstein O, et al. Meta-analysis: the relative efficacy of oral bowel preparations for colonoscopy 1985-2010. Aliment Pharmacol Ther. 2012;35:222-237.

5. Sint NJ, de Jonge V, Korfage IJ, et al. Benchmarking patient experiences in colonoscopy using the Global Rating Scale. Endoscopy. 2012;44:462-472.

6. Hassan C, Bretthauer M, Kaminski MF, et al. Bowel preparation for colonoscopy. European Society of Gastrointestinal Endoscopy (ESGE) Guideline. Endoscopy. 2013;45:142-155.

7. Calderwood AH, Jacobson BC. Comprehensive validation of the Boston Bowel Preparation Scale. Gastrointest Endosc. 2010;72: 686-692. 